Voix et Images

volxetimages

\title{
Rodolphe de Repentigny : critique d'art, théoricien et artiste
}

\section{Marie Carani}

Volume 10, numéro 3, printemps 1985

André Major

URI : https://id.erudit.org/iderudit/200520ar

DOI : https://doi.org/10.7202/200520ar

Aller au sommaire du numéro

\section{Éditeur(s)}

Université du Québec à Montréal

\section{ISSN}

0318-9201 (imprimé)

1705-933X (numérique)

Découvrir la revue

\section{Citer cet article}

Carani, M. (1985). Rodolphe de Repentigny : critique d'art, théoricien et artiste. Voix et Images, 10(3), 137-151. https://doi.org/10.7202/200520ar d'utilisation que vous pouvez consulter en ligne.

https://apropos.erudit.org/fr/usagers/politique-dutilisation/ 


\title{
Rodolphe de Repentigny: critique d'art, théoricien et artiste
}

\author{
par Marie Carani, Université du Québec à Montréal
}

Dans les milieux intellectuel et artistique des années 1940-1950, les enjeux esthétiques et éthiques étaient constamment véhiculés par des confrontations entre les tenants de «l'académisme» et ceux de "l'art vivant». À cette époque, la figuration académique et conventionnelle était encore la seule forme de peinture acceptée officiellement par les autorités publiques, les musées, les galeries, et le public habituel des manifestations plastiques. C'est dire que le contexte duplessiste n'encourageait pas une recherche picturale non-figurative, et qu'aux débuts des années 50, la figuration triomphait encore et contrait toute autre forme de recherche picturale. Le conservatisme idéologique et politique du régime duplessiste s'exprimait ainsi en art par l'assimilation et l'apprentissage d'une technique, d'une formation perçue comme «sérieuse»: une telle valorisation de «l'académisme» réalisait dans le domaine culturel, comme l'a déjà noté François-Marc Gagnon, les propositions de «l'idéologie de conservation», pour le maintien de l'Ordre politico-religieux établi1. Rodolphe de Repentigny refusa publiquement un tel statu quo.

La résistance à cette "Grande Noirceur» se manifesta en peinture par l'affirmation d'une pratique non-figurative automatiste, puis plasticienne. La lutte pour imposer cette peinture "abstraite» fut assurément le principal enjeu esthétique du milieu artistique québécois de l'époque. Dans ce débat, l'influence de P.-E. Borduas s'avéra déterminante. Sa prise de la parole, donc de la liberté dans le milieu social, son Refus Global, son rejet de tous les "poncifs» de l'art académique figuratif au nom d'une peinture nonfigurative exprimant "sa sensibilité à lui 2 », tracèrent, pour les résistants intellectuels et artistiques n'ayant pas abandonné le combat contre le pouvoir et le "goût» officiel, une voie exemplaire. R. de Repentigny inscrivit ses pratiques, ses problématiques, ainsi que son insertion sociale dans le creuset de cette rupture qu'il qualifia lui-même de «germinale» dans le Manifeste des Plasticiens ${ }^{3}$. Intellectuel, critique d'art, théoricien, peintre et photographe, il anima d'une manière critique le milieu artistique québécois au même titre que Borduas l'avait fait durant les années 40 .

En tant que défense de l'art non-figuratif, des recherches automatistes et abstraites-géométriques, et intervention dans les débats philosophiques et esthétiques de l'époque, la critique de Repentigny devint un critère d'excellence pour l'ensemble du monde de l'art, comme en témoignèrent les affrontements qu'elle suscita régulièrement avec les autres critiques. Pendant toutes les années 50, ses commentaires furent lus et discutés comme des points de mire nécessaires à une véritable compréhension des événements et manifestations artistiques. Théoricien du mouvement des Premiers Plasticiens qui succéda à l'Automatisme comme pôle d'attraction de la jeune peinture québécoise, Repentigny impose par le dynamisme de sa réflexion 
une nouvelle tendance picturale qui fondait sa démarche sur la philosophie existentialiste et sur la psychanalyse, ce qui proposait une structuration renouvelée de l'espace pictural, un espace «plasticien». Sous le pseudonyme de Jauran, Repentigny actualisa dans une pratique plastique un rapport entre théorie et pratique de nature essentiellement existentialiste, basé sur la nécessité pour une crịtique d'art véritable de «s'assumer» dans une ouvre.

C'est le triple rôle de critique d'art, de théoricien du plasticisme et d'artiste, assumé par Repentigny, unique dans le milieu intellectuel et artistique québécois, que nous voulons circonscrire ici en articulant ses principaux fondements esthétiques et philosophiques.

Dans un contexte social peu enclin à «s'ouvrir» aux visées de la pratique picturale, Rodolphe de Repentigny assura dans le quotidien la Presse (de 52 à 59), dans l'hebdomadaire progressiste l'Autorité du Peuple (de 53 à 55), et dans la revue Vie des Arts (de 56 à 59)4, une couverture exhaustive de l'évolution et du développement des pratiques plastiques, tout en formulant une réflexion structurée sur les rapports entre l'art et la société. En moins de dix ans, ce jeune homme qui n'avait que 33 ans lors de son décès prématuré survenu en 1959 (à la suite d'un accident d'alpinisme), élabora un corpus critique de plus de 1000 textes où il mena une lutte constante contre l'obscurantisme officiel. Au point que sa disparition fut vécue comme une "catastrophe» par ses contemporains. Coïncidence troublante, son décès précéda de très peu celui de Maurice Duplessis, l'adversaire contre lequel il s'était le plus souvent élevé. On peut considérer à juste titre que le militantisme de son action culturelle ne fut pas étranger à l'effritement progressif vécu par le duplessisme au cours des années 50, et qu'il généra même plusieurs des bouleversements politiques et culturels revendiqués par la Révolution tranquille.

Ses écrits critiques, publiés régulièrement deux, trois, ou même quatre fois par semaine, entre 52 et 59 , imposèrent d'emblée Repentigny comme le «chien de garde» de son milieu. Face à un public peu informé des problématiques picturales aussi bien figuratives que non-figuratives, Repentigny se refusa à toute censure de ses opinions. Engagé à la Presse au poste précaire du «chroniqueur artistique 5 », il ne put accepter de taire son enthousiasme et sa sympathie pour les mises en question de l'académisme, malgré les mots d'ordre d'ignorer l'existence même des recherches nonfiguratives qui remontaient pourtant déjà aux premières gouaches de Borduas de 1942.

Repentigny concevait son travail comme une fonction socialement responsable, et à cette fin, comme il l'affirma lui-même à plusieurs reprises, pour compenser l'inaction des milieux éducatifs, la critique d'art se devait de guider et d'orienter le public en favorisant l'ouverture d'un dialogue, un contact nécessaire entre celui-ci et l'œeuvre d'art. Par des articles d'information sur les différentes expositions et manifestations de l'art abstrait na- 
tional ou international, par des articles de fond sur une tendance ou un artiste, par la reproduction constante (après l'été 53) de ces œuvres qui avait jusqu'alors été interdite dans un quotidien comme la Presse, par ses bilans annuels (critiques, statistiques) sur la progression des pratiques abstraites, et enfin par la place prioritaire qu'il lui accordait dans son calendrier hebdomadaire des événements artistiques, Repentigny actualisa dans sa critique une défense exemplaire et toujours exigeante de cet art.

Repentigny interprétait la résistance devant l'art abstrait comme la démonstration d'un manque de formation visuelle, d'un "défaut» dans l'éducation artistique. Par sa critique d'art, il voulait remédier à cet état de choses. La constatation suivante sur la nature du rapport entre l'éducation et la perception indique bien le sens didactique de ses critiques: «C'est à cause d'un défaut dans leur éducation si tant de gens sont prêts à dire, devant les ouvres contemporaines, «je pourrais faire la même chose», entendant par là que la "chose» est sans le moindre intérêt 6 ». Ce travail d'information concerna surtout les problématiques de l'abstraction.

R. de Repentigny s'intéressa dès son entrée au journal la Presse aux pratiques des automatistes montréalais. Il consacra son premier article sur l'art abstrait aux travaux des jeunes automatistes Robert Blair et Marcelle Ferron, disciples de Borduas. Dans «Des peintres qui ne voient pas à travers les fenêtres", publié en novembre 52, il traçait ainsi les acquis de la nonfiguration «bidimensionnelle»: «(Robert Blair) ne peint jamais derrière le mur, mais devant (...) Il n'y a aucune dimension dans ses tableaux. On peut les renverser, les couper en deux, en quatre, ce serait du pareil au même (...) Les peintres qui adoptent ce genre le font par choix, et (...) ils pourraient, s'ils le voulaient, peindre des fenêtres dans le mur. Par bonheur, ils ne le font pas. Par bonheur, parce que nous en avons déjà pas mal de fenêtres 7 ». Il est à noter qu'en 52, l'espace automatiste était encore perçu comme bidimensionnel, et non comme il le sera plus tard, par Borduas lui-même dès son exil new-yorkais de l'automne 53, comme un espace cubiste rapproché. C'est cette interprétation initiale que Repentigny véhiculait dans son premier article sur l'abstraction. Cependant, comme Borduas, il en vint progressivement à remettre en question la bidimensionnalité de cette peinture, ce qui l'amena en 54 à formuler une structuration spatiale "autre», qui se voulait véritablement bidimensionnelle, soit un espace «plasticien» abstrait et géométrique.

Nonobstant ces préoccupations, Repentigny salua l'exposition automatiste "La matière chante» tenue en avril-mai 54 comme une manifestation de "première importance» et comme la preuve de la "vitalité» de l'art "créateur» au Québec8. C'est qu'il interprétait les tableaux exposés comme des expressions privilégiées du principe de la «nécessité intérieure» défini par Kandinsky dans Du spirituel dans l'art, comme le garant de l'authenticité, de la sincérité et de l'honnêteté de l'artiste créateur9. Par cette référence aux valeurs d'authenticité de l'être 
créateur, Repentigny définissait son attachement fondamental aux valeurs de l'existentialisme.

Il publia aussi le 1 er mai 54 dans l'Autorité, un article sur ces ouvres automatistes, intitulé "Comment faut-il regarder une toile non-figurative?», où il affirmait sa conviction que le spectateur devrait abandonner toutes «prétentions rationalistes» pour s'investir totalement, existentiellement, de façon non-verbale, dans ces productions non-figuratives, en admettant "que le peintre nous dépasse», et que «la part de l'intelligence est surtout de nous donner devant les oeuvres d'art une conduite qui rende disponible notre sensibilité10".

En démarquant sa perception de l'ouvre d'art non-figurative des formes de représentation figurative traditionnelles et de la littérature sur la peinture, Repentigny articulait son principe d'une connaissance «intuitive» de l'œuvre plastique comme critère générateur de la production et de l'interprétation de l'art. Le trajet existentiel «expérimenté» dans la production artistique définissait son caractère de démarche individuelle et collective essentielle pour une re-découverte des «nécessités», de ce que Repentigny entendait dans le.Manifeste des Plasticiens par une peinture "d'ultimes nécessités, d'inévitables obsessions, de réductions transcendantales 11 ». En ce sens, la production artistique fut toujours perçue par Repentigny comme une recherche. L'impression généralisée que cette activité ne serait qu'un agréable "passe-temps» était à l'opposé de sa conception de l'art comme "action", comme pratique créatrice existentialiste et transformation des valeurs.

La démarche artistique se devait d'être une aventure où l'artiste et le spectateur étaient impliqués dans une recherche de la "vérité». Quand Repentigny déclarait que «la peinture est un langage direct, une action» qu'il oppose à l'expression verbale, «simple traduction de la pensée et de la perception 12», il proclamait que la pratique de l'art était une expérience individuelle s'identifiant au processus de la création: "La création est l'authentique réalisation d'un être unique (...) La création picturale s'accompagne de l'entrée dans une vie nouvelle, un monde libre, ne dépendant idéalement que de la :personne13». Par l'art, l'artiste réaliserait ainsi «la vérité, sa vérité14», et assumerait sa liberté, ce qui nécessiterait cependant sa «soumission» aux valeurs fondamentales d'authenticité.

En tant qu'existentialiste, Repentigny considérait d'ailleurs «l'expérience» comme un acte psychique de «plaisir», comme l'acte psychique individuel fondamental. Il se référait au "principe de plaisir» proposé par Freud comme le fondement épistémologique des actions humaines, en lutte contre les valeurs de la «raison». C'est d'ailleurs ce que Repentigny exprima dans le Manifeste des Plasticiens en définissant la peinture plasticienne comme le visage «d'ultimes nécessités» ne répondant pas à des «choix» ou des a priori rationnels, mais faisant appel au niveau de connaissance de "l'intuition» opposé à celui de la «science15». Cette valorisation de la fonction psychanalytique dans le système de la connaissance, soit l'introduction du niveau des «nécessités» d'un être totalement «libre», non asservi à toute in- 
tentionnalité, ést assurément reliée aux valeurs de l'inconscient défendues par l'Automatisme surrationnel, notamment par Borduas dans le Refus Global. L'influence de ce premier refus de «l'esprit utilitaire» transformant l'homme en être uniquement «productiviste», est manifeste dans la réflexion de Repentigny.

L'intrusion de la dialectique freudienne dans «l'expérience» de l'art, faisant suite au procès «d'intuition-création» de l'artiste, posait les balises de la fonction de l'art. Pour Repentigny, l'art se devait "d'assumer son temps», (de) le dépasse(r) même, en le sublimant 16» par sa re-découverte de la liberté primale, dégagée des contraintes et des asservissements imposés par les valeurs collectives. C'est dire que la notion de plaisir-liberté constituait une coupure épistémologique dans les rapports de l'individu avec la société, et que cette rupture fondamentale était véhiculée, sublimée, par la fonction artistique. Repentigny proposait donc à l'art de «tendre vers le cosmos» par-dessus les «drames individuels»: dans la re-construction des rapports d'intégration de l'art et de la vie, permise par l'expression sublimée des pulsions instinctives de la libre création 17. La seule démarche exploratoire devant les productions non-figuratives serait de «taire son esprit et de se livrer silencieusement au tableau 18 ».

Le trajet existentiel et l'angoisse qui en résultait, étaient pour Repentigny le champ privilégié de la production plastique. L'ouvre d'art se posait comme la nouvelle symbolique de la "recherche angoissée» de l'individu pour sa «vérité». En ce sens cette quête artistique pouvait être interprétée comme une poursuite "de l'absolu», qui impliquait une "communion» dans l'expérience de l'art 19. Cette "émotion durable», comme la qualifie Repentigny, était bien une réponse au phénomène d'angoisse devant «l'insuffisance du monde». Dans les Aphorismes du Manifeste des Plasticiens, il ajouta que ce trajet représentait la "spiritualité» de notre époque: "Il ne reste de spirituel que l'angoisse. Il n'y a pas en 1955 d'art sacré: l'art est sacré20». Ce faisant, il manifestait une volonté d'inscrire les symbolismes picturaux dans les schèmes d'une psychologie existentialiste, fondée sur la nécessité pour le créateur et pour le regardeur d'assumer leur époque, de transgresser l'angoisse de vivre par cette nouvelle symbolisation vitale, pardelà le combat que chaque être devait mener avec ses contingences personnelles. Et le rôle essentiel de la critique d'art était «d'exiger» qu'il en soit ainsi.

Le sens de l'exigence posée par Repentigny à la pratique de la critique d'art entraînait aussi la responsabilité pour le critique «d'assumer». sa liberté. D'ailleurs lorsqu'un lecteur lui reprocha de ne pas «s'engager» à fond dans une défense inconditionnelle de la peinture vivante, de ne pas s'impliquer d'une façon nettement apologétique en faveur de cette seule peinture, Repentigny lui répondit que son adhésion aux valeurs de l'abstraction ne pouvait éliminer sa liberté critique de refuser un simple travail de promotion de cet art: «Ne pas exprimer publiquement les réserves sur certains peintres, cela revient à demander que la critique ne soit que promotion, propagande. Une suspension de la critique au nom d'intérêts 
supérieurs, c'est faire de la politique, tout simplement 21 ». Impliqué comme il l'était dans la défense active de l'art abstrait, il ne se résolut cependant jamais à faire de son métier un exercice assimilable à de «la politique».

Repentigny ne se limitait pas à un strict discours sur la pratique artistique, dans le cadre d'un médium de communication de l'art, mais il concevait aussi sa production critique comme une "action", une pratique de transformation des rapports de l'homme au monde. En intervenant d'une façon agissante dans son milieu, il voulait élaborer une éthique sociale, une «re-prise de conscience». Sa critique des institutions du milieu de l'art, des rôles joués par l'État, les musées publics et privés, les galeries, les collectionneurs dans la diffusion et la "commercialisation» de l'art, se voulut constamment un questionnement du détournement de sens mis en ouvre par ce niveau «de distribution» des productions plastiques. Cette perte de contrôle par l'artiste du "destin de l'œuvre» ne pouvait résulter selon Repentigny qu'en une négation complète de ses valeurs inhérentes de transformation sociale. C'est pourquoi il prôna un renouvellement du rôle des artistes euxmêmes dans le procès de diffusion artistique. Dans un Musée de l'art vivant à construire, un Musée d'art contemporain où les artistes dirigeraient et organiseraient, sans intermédiaires leurs propres expositions, on pourrait assister à une véritable confrontation des regardeurs avec les problématiques non-figuratives chargées de signifiants «autres». Il est à noter que Repentigny fut l'un des premiers au Québec à réclamer publiquement l'ouverture d'un tel musée voué exclusivement à l'art actuel.

Dans la dialectique repentignienne, en investissant le présent, l'art créateur définit ce qui paraîtra dans l'avenir une nécessité vitale pour l'humanité. Les créateurs actuels œuvrent à la re-définition d'une «communauté de valeurs»; car, malgré leur isolement d'artistes ne travaillant "pas pour un public, ni pour une entité sociale, mais pour l'art luimême22», ces créateurs élaborent des valeurs auxquelles «adhéreront» des membres de la société ayant accompli une démarche similaire, ou sentant «le besoin» de le faire. C'est par cette exemplarité que les créateurs «prendront ainsi l'allure de prophètes 23 ", donc de précurseurs du renouveau sociétal, ou de "phares» au sens baudelairien.

Dans «Pour nous apprendre à voir», Repentigny identifiait ce processus comme une "métamorphose» spirituelle (au sens d'André Malraux 24), une transmutation imaginaire de notre ambiance physique, de son matérialisme «fonctionnel et productiviste»: il y affirme que la fonction principale de l'artiste n'est pas de «niveler» physiquement les «accidents de la nature» pour construire "des paysages d'acier», mais de transformer «spirituellement» l'ambiance urbaine des sociétés modernes en donnant à la collectivité humaine «une nouvelle vision de ce qu'elle (a regardé) hier25». Ce processus de regénérescence vitale de la société, Repentigny le qualifia "d'essentiel à la vie». Il en fit le fondement d'une «métamorphose sociale».

À une époque où «les problèmes humains reçoivent des solutions collectives, conçues et réalisées collectivement 26 », les apports gratuits de la 
liberté individuelle permettraient la dissolution des valeurs «mécaniques» actuelles. Devant sa «constatation du décès de la culture européenne (...) aplanie par le machinisme», devant la définition strictement "utilitariste» de la vie dans une société "matérialiste», devant un tel ravalement des valeurs humanistes, Repentigny proposait l'intrusion du «nouvel humanisme» malraucien sublimé dans le rapport au monde vécu par l'artiste créateur. Dans «L'art est aussi lien avec le monde», il voit dans ce travail un effort de communication, l'ouverture d'un dialogue avec la société mécanique, "œuvre de la science», et une façon "d'humaniser ce monde technique» en projetant plastiquement «les états d'esprit qu'il provoque». Il poursuit même que ce n'est pas «en fuyant vers les verts bosquets et les petites routines» que cette société pourra être maîtrisée, mais en l'affrontant sur son propre terrain27. Un retour à la nature, donc à une société pré-industrielle, ne serait pas une solution «assumant» existentiellement son milieu de vie, car celui-ci est «depuis longtemps» un monde résolument urbain et machiniste: «Notre vrai milieu vital, ce n'est plus la verdoyante nature, depuis longtemps, mais bien la scintillante et bruyante ville. Les véritables autruis non-humains sont les machines avec lesquelles nous avons un quotidien contact, qui «vivent» pour nous et par lesquelles nous vivons 28 ").

En assumant ce que Sartre nommait la «situation fondamentale» de son époque, l'artiste, bien qu'il ne soit pas un «révolutionnaire» politique puisqu'il a rompu avec toute idéologisation de l'art, n'en réaliserait pas moins une dissidence et une transgression d'ordre éthique beaucoup plus fondamentale: tout en subissant cette «situation fondamentale», il ne s'avoue jamais vaincu, mais «feint d'accepter», et en ce sens il actualise une production «essentiellement subversive 29 ». Par cet acte d'insubordination enraciné dans le milieu de vie des créateurs, on assisterait à un ressourcement des fondements philosophiques et épistémologiques de la société: «Nous vivons dans une grande ville industrielle, au climat âpre (...) Il faut comprendre qu'il ne peut être ici question de révolte (...) Pour s'élever contre la machine, il faut avoir l'âme réactionnaire - ce dont nous n'avons nul besoin en ce moment. La seule attitude qui permette de survivre est celle de l'homme qui s'annexe la machine30». Et la critique d'art qui est "action» se devrait d'ouvrer à réfuter et dénoncer toute velléité de démission des artistes "devant leur's responsabilités d'hommes vivant en société31».

Une résistance sociale se dressant contre ces efforts de changement ne serait d'ailleurs pas anormale selon Repentigny: au contraire, «il faudrait s'inquiéter si, dans une société généralement admise comme matérialiste, les arts plastiques s'incorporaient tels qu'ils se présentent et sans obstacle à la vie courante32». Et c'est pour contourner ces «obstacles» que la critique d'art véritable se devrait d'intervenir constamment dans son milieu par une action éducative et pédagogique visant à communiquer «l'expérience»artistique à l'ensemble de la société.

Dans la pensée de Repentigny, la fonction sociale de l'art n'est jamais autoritaire, programmatique, mais résolument égalitaire, démocratique; en 
fait il prônait une démocratisation de l'art qui l'amena aussi à reconnaître les valeurs fondamentales d'authenticité de «l'art populaire», de l'art «des peintres du dimanche». Il y voyait le véhicule d'une contestation permanente de tous les ordres établis. Sa vision sociale s'inscrivait d'emblée dans un processus imaginaire de transgression, qui ne serait pas socialement réprimable ou récupérable.

Quand Repentigny proclama dans «Méditation sur l'actualité de l'art à Montréal» que les débuts du peintre Fernand Leduc comme critique, étaient une signe que «la critique est une action, aussi intense que (sa) peinture», et que cela «déprécie du fait même tout dilettantisme», au point que dans quelques temps «plus personne ne voudra écouter ceux qui ne nourrissent par une "oeuvre", ceux dont les paroles ne sont que des coups de chapeau $33 »$, il énonçait la nécessité pour un critique de s'actualiser, de se réaliser au-delà des mots dans une pratique plastique. Il posait que «la recherche d'une réalité humaine ayant une multiplicité de dimensions», déprécie tout «dilettantisme» et légitime au contraire son praticien en raison de «l'intensité» de l'aventure existentielle qu'elle affirme. Repentigny présentait donc la double fonction du peintre-critique comme l'expression totale du drame existentiel, le seul garant de sa sincérité, et de facto de sa légitimité sociale. Bien plus qu'une simple vérification de la théorie soustendant l'œuvre, la pratique deviendrait en regard de cette conception le lieu même d'affirmation, de confrontation, de la valeur juste et véritable d'une critique d'art, le moyen vitaliste par excellence d'une critique conçue comme «action».

Dans «Gare aux néo-académismes», il précisait que cette conception opposée à ce qu'il nomme «l'énoncé de principes», définissait que c'était bien «sur la toile, ou la masonite, que les créations picturales trouvent leur naissance et non dans les encriers $34 »$. Conséquemment il fondait la démarche picturale sur le modèle bergsonien d'une primauté absolue accordée à «l'intuition». C'est ce qu'il inscrivit dans le Manifeste des Plasticiens comme fondement de la pratique picturale du groupe. (On les nomme aujourd'hui les Premiers Plasticiens par opposition aux Seconds Plasticiens: Molinari, Claude Tousignant, Juneau, Goguen). Ce groupe des Plasticiens, comprenant Jauran (R. de Repentigny), Louis Belzile, Fernand Toupin et Jean-Paul Jérôme, actif entre 1954 et 1956 et qui devait supplanter le mouvement automatiste et post-automatiste dans le rôle de moteur et de pôle d'attraction de la jeune peinture québécoise à partir du milieu des années 50 , était directement issu de la théorisation repentignienne d'une telle peinture «intuitive» qui ne serait pas le produit d'un rêve «romantique», donc d'un «subjectivisme pictural35» comme l'Automatisme, mais plutôt l'expression d'une problématique spatiale d'ordonnance abstraite, géométrique.

Animateur et théoricien de ce mouvement des Premiers Plasticiens, Repentigny rédigea et fit publier lors de la première exposition solo de ces peintres à l'Échourie en février 55, le Manifeste des Plasticiens. Il y définissait une esthétique de l'abstraction, où les notions d'un art abstrait y 
étaient tributaires des thèses défendues en France durant les années 50 par le Groupe Espace, composé d'artistes et de critiques tels Michel Seuphor, Roger Bordier, André Bloc, Vasarely. Ce groupe ouvrait à l'intégration des arts de la peinture et de l'architecture, et reprenait les problématiques d'équilibre relationnel des éléments formels et d'espace non-mimétique du groupe européen Cercle et Carré36.

En novembre 54, au moment de la huitième exposition collective de la librairie Tranquille où étaient exposés entre autres les travaux de jeunes abstraits comme Jauran et Belzile, Rodolphe de Repentigny avait déjà défini dans la Presse la production de ces peintres, qu'il qualifia alors pour la première fois de "Plasticiens», comme «l'emploi de formes géométriques, dans des compositions abstraites37». Dans l'Autorité, il avait aussi rédigé à cette occasion un article illustré, intitulé «Après les automatistes et les romantiques du surréalisme: les Plasticiens», où il formulait la définition suivante de leur travail: «Ils mettent l'accent sur l'aspect "plastique» de la peinture, sur son autonomie formelle $38 »$. Il y posait les premiers jalons d'une conception «plasticienne» de la peinture: une œuvre abstraite, aux formes géométriques, constituée d'éléments plastiques totalement intégrés à la surface de la toile, au rythme "fonctionnel». Quant aux rapports formels des éléments, il les décrivait ainsi: «Chaque tableau a un «ton» bien particulier, une harmonie de couleurs dont l'aspect complémentaire est l'harmonie des formes». Ces propositions avançaient la nécessité formelle d'un équilibre relationnel des éléments de formes et de couleurs.

Le Manifeste des Plasticiens, publié le 10 février 1955, devait concrétiser l'ensemble des positions de Repentigny sur la peinture. Son système esthétique y est globalement articulé, dans ses fondements philosophiques et plastiques. Il s'agit d'une synthèse intégrant ses valeurs fondamentales existentialistes, ses notions philosophiques sur l'intuition-création, l'intuition intuitionnée, et la vérité, à des principes et critères plasticiens de production, et par le fait même d'analyse critique de l'ouvre d'art.

La constitution d'une "peinture plasticienne» se situait pour Repentigny dans le cadre d'une volonté d'abandon de toutes références figuratives, pour privilégier l'organisation picturale des éléments plastiques. Le développement d'un tel art dégagé de toutes considérations littéraires, refusant par le fait même toutes notions extérieures à la plasticité, posait un «espace proprement pictural». Aux formules et conventions visuelles de la peinture figurative, c'est-à-dire sa manipulation de l'objet tridimensionnel selon ses valeurs illusionnistes de profondeur perspectiviste, de gravitation et de volume, traduisant une nature intentionnelle pré-conçue et préordonnée selon un système de représentation réaliste, Repentigny opposait la réalisation spatiale d'un nouvel ordre anti-illusionniste ${ }^{39}$. Il se refusait à toutes «concessions» face aux représentations conventionnelles.

Toutes considérations littéraires poétiques ou oniriques, tout contenu symbolique servant à mettre en évidence tel aspect référentiel du tableau se trouvaient alors éliminés au profit d'un art expressif ne se référant qu'aux éléments plastiques du tableau et à leur effet émotif. D'ailleurs la recherche 
repentignienne d'un «équilibre» relationnel des éléments formels, où la nécessité plastique est issue de l'émotion, définissait et proposait la construction d'un objectivisme pictural exprimant un «équilibre interne», très "vital». C'est dire que la nouveauté qu'il recherchait dans la forme, il la recherchait aussi dans l'émotion, ce que ne reconnurent pas les adversaires de cette peinture qui la qualifiaient de "froide», de «désincarnée», voire même de simple produit d'un «calcul rationnel». Une telle réception critique fut généralement partagée par la plupart des intervenants du milieu de l'art des années 50, comme le démontre justement le sobriquet de "calculateurs géométriques» que le critique René Chicoine leur attribua dans le Devoir. Repentigny formulait la réalisation d'une «pure problématique plastique» de "détermination de forme» abstraite géométrique, évacuant toute référence à ce qu'il nomme «l'émouvant». La recherche de l'expression passait donc pour les Plasticiens par l'élaboration d'un ordre structurel universel fondé sur une stricte plasticité transcendant les «drames intimes»: c'est bien ce qu'entendait Repentigny lorsqu'il affirmait que «l'art (abstrait) tend vers le cosmique, par-dessus les drames individuels, en recherchant les sources mêmes de l'expression 40 \%.

Repentigny avançait dans le Manifeste de 55 une série de propositions sur le sens et la portée du travail pictural des jeunes Plasticiens. Il indiquait tout d'abord la raison d'être du groupe: il n'était pas issu d'une volonté d'application ou de promotion d'une idée, d'une théorie préconçue, donc d'une intention préalable, mais d'un recoupement similaire dans leurs pratiques picturales (se produisant à la suite de quelques expositions collectives chez Tranquille durant l'été et l'automne 54), qui dénotait un même attachement prioritaire aux seuls faits plastiques, et aux rapports de ces éléments picturaux, "éléments assumés comme fins». Ces faits plastiques retenus comme définissant «ce qui est particulier à la peinture», c'étaient les «ton, texture, formes, lignes, unité finale qu'est le tableau, et les rapports entre ces éléments $41 »$.

Repentigny identifiait ensuite la pratique des Plasticiens comme un «épurement incessant» des éléments plastiques présidant à leur arrangement formel. Leur «destin», la «révélation des formes parfaites dans un ordre parfait», était alors la réalisation d'un ordre idéal. Mais cet ordre ne pouvait se constituer que dans «l'amour du moment présent», car c'est seulement par la pratique individuelle de la création libre immédiate, que ces peintres pouvaient atteindre à leur vérité intuitive. Cette conception de la liberté individuelle, totale, du travail de création posait aussi l'impossibilité de toute découverte d'une "vérité objective» collective, la peinture plasticienne n'étant pas l'illustration d'une idéologie, mais uniquement une expression de rapports «existentiels» non asservis.

Les Plasticiens devaient élaborer uniquement des objets autonomes, c'est-à-dire qu'au tableau conventionnel, tridimensionnel, devait succéder un tableau spécifiquement attaché à l'organisation des différents éléments de sa surface, ce qui le fonderait comme un «objet plastique». Le tableau ne serait plus ce plan transparent qui tronquait la pyramide visuelle, ce «trou 
dans le mur», mais bien un objet autonome d'une telle représentation du monde. Et ces «tableaux-objets" spécifiquement picturaux seraient des «totalités peintes» aux structures de composition intégrées et ordonnées selon un structuration organique, fonctionnelle, tributaire d'un modèle vital biologique et naturaliste, dont chaque élément participe au rythme de l'ensemble.

Dans les derniers paragraphes du Manifeste, Repentigny précisait les relations des Plasticiens avec l'histoire de l'art. Il notait tout d'abord, reprenant les propos d'André Malraux dans les Voix du silence, que «les tableaux naissent des tableaux ${ }^{42}$ ». Les productions picturales du groupe n'étaient donc pas nécessairement tout à fait nouvelles. Au Canada, et au Québec, il posait l'influence "germinale» de la "révolution automatiste amorcée par Borduas». Plus généralement, dans l'histoire de la peinture, il reconnaissait l'existence d'une suite de libérations artistiques dans le cadre d'évolution de la civilisation chrétienne, et cela en vertu d'une conception cyclique de l'art qu'il avait repris de Roger Bordier du Groupe Espace43. L'histoire de l'art serait l'actualisation de trois grandes étapes de libération. La libération du «rituel spirituel» avec la Renaissance: car alors l'art «s'humanise» après avoir été «au service» d'un rituel religieux, c'est-à-dire un «art sacré» voué à l'identification de l'humanité. Malheureusement les arts renaissants n'ont alors qu'un rôle «mondain» de glorification, de flatterie «de l'élite de la puissance», d'où un nouvel asservissement «matérialiste» qui sera dénoncé par la libération du «rituel matérialiste» mis en oeuvre aux XIXe et XXe siècles par les mouvements impressionniste, fauviste, cubiste, dadaïste, et surréaliste et qui formulent une «re-prise de conscience des valeurs artistiques», voire même un questionnement de la notion de "chef-d'ouvre». C'est d'ailleurs à ce niveau qu'intervenait l'Automatisme surrationnel montréalais et ses valeurs de l'inconscient qui permirent, selon Repentigny, la libération du peintre des contraintes officielles au profit de l'expression affective, «romantique» de son moi. Cependant après cet «interlude» de la "peinture poétique», une dernière libération, du moins partielle, celle de la peinture assumée comme fin en elle-même, dégagée d'une simple expression "du moi», véhiculée par les travaux «des tendances abstraites de la peinture contemporaine, soit-elle tachiste ou plasticienne», impose les problématiques des matériaux eux-mêmes, de la plasticité, sans justification extérieure aux seuls faits plastiques 44 .

Pour Repentigny, avec cette épuration des «apparences justificatrices» permises par les tendances abstraites, l'ensemble du processus cyclique était terminé, l'ultime «aliénation de l'œuvre peinte» ayant été vaincue par l'abandon de «la concentration sur lui-même» de l'artiste. "Tout ce qui était émondable ayant été émondé45», notamment avec les recherches de Piet Mondrian d'un «équilibre pur» des éléments plastiques. Le Néo-Plasticisme de Mondrian, comme pratique abstraite paradigmatique, rompait avec l'espace illusionniste d'une représentation naturaliste romantique. La peinture de Kandinsky tendait encore à ramener l'être aux 
«rythmes de la nature». C'est aussi le cas de la peinture automatiste québécoise, et la pensée de Mondrian devait conséquemment avoir une influence déterminante sur la réflexion de Repentigny46. Quand Mondrian formulait la nécessité d'un rapport entre peinture néoplasticienne et ambiance physique, dans «Le home - la rue - la cité 47 », il générait un courant de pensée qui influença Cercle et Carré, le Groupe Espace et aussi R. de Repentigny dans sa formulation d'une peinture plasticienne s'annexant l'ambiance urbaine et «la machine», le mur et l'édifice, etc...

Dans les Aphorismes plasticiens accompagnant le Manifeste, Repentigny énonçait des mots d'ordre plastiques et perceptifs découlant de cette esthétique. On y retrouve ses conceptions de la «vérité», de «l'intuition», de «l'intuition intuitionnée» permettant au regardeur de savoir qu'une cuvre est «vraie pour son créateur», ce qui est «l'amour du prochain», voire «l'existence de l'autre».

Ce sont ces valeurs «plasticiennes» inscrites dans le Manifeste que Repentigny-Jauran voulut assumer dans sa propre production picturale. Comme peintre plasticien, il ceuvra de l'automne 53 au printemps 56, et poursuivit par la suite cette pratique artistique dans une production photographique plutôt centrée sur une exploration des potentialités du médium.

Chez Repentigny, au niveau du rapport discours/peinture, on retrouvait la situation suivante: autant comme critique théoricien du plasticisme ou peintre, il formulait une esthétique picturale dont l'équilibre relationnel était fondé sur une structure de composition all-over, nonhiérarchisée, intégrée, de la surface. Cette peinture qui, par définition même, en était une de «détermination de forme», n'accordait de facto qu'un rôle complémentaire à la couleur. Repentigny favorisait généralement l'emploi de couleurs tonales, «assombries», aux valeurs rapprochées, donc dans des contrastes de valeurs plutôt faibles. Cette peinture de tons et de demi-tons étudiés refusa ainsi initialement les couleurs pures, vives, perçues comme «exclamatives», c'est-à-dire comme des ruptures de l'équilibre recherché. Progressivement cependant, à partir de 1956, Repentigny fut plus réceptif aux couleurs pures, aux contrastes plus accentués, pourvu qu'ils soient inscrits dans des équilibres relationnels harmonieux.

Parti d'un espace tridimensionnel rapproché, hérité du cubisme dans la filiation de l'Automatisme où des masses focalisées étaient organisées en relations formelles et chromatiques par superposition et enchâssement, il chercha à s'en libérer par l'affirmation d'une surface bidimensionnelle. Pour ce faire, il multiplia tout d'abord les centrations pour occuper toute la surface grâce à un processus de réduction et de multiplication des formes. Puis inversement, dans une volonté d'imposer une bidimensionnalité de la surface, il réduisit et agrandit ces formes, et les structura par une juxtaposition séquentielle associée à une superposition et un enchâssement, tout en conservant une centration -focalisée. Cette évolution culmina-dans un assemblage séquentiel devenu prédominant, où on retrouvait un espace allover non vectoriel. 
Au niveau des rapports chromatiques, la peinture de Jauran présenta une évolution encore plus marquée. Alors que l'ensemble de sa production avait été caractérisée par une utilisation de teintes traitées en valeurs, souvent dans des harmonies sombres à la manière de la palette automatiste, où le peintre travaillait à des rapports de masses claires et sombres, aux contrastes réduits, Jauran introduisit en 1956 la couleur pure, éliminant les tonales, mais en encadrant tout de même les possibilités énergétiques de la couleur, et l'affirmation de contrastes trop accentués, en cernant ses plans de couleur pure par des traits noirs fortement marqués ou des réserves.

On peut donc voir dans sa peinture une pratique plastique cherchant à générer les mêmes valeurs que celles véhiculées dans sa critique d'art et dans ses réflexions théoriques sur la peinture. En s'insérant initialement dans le courant cubiste de manipulation de l'objet tridimensionnel, Jauran démontra une volonté de se dégager de ce type d'espace pour réaliser spatialement ses valeurs esthétiques.

Le Manifeste avait posé l'ensemble de la conception repentignienne d'une pratique picturale contemporaine devenue avec l'abstraction, " $u$ une action qui a son propre sens 48 ». C'est cette signification auto-référentielle qui terminait le cycle des révolutions de la peinture. La peinture plasticienne était «abstraite» par définition même car elle avait désubjectivisé l'objet pictural, et pour cela était «axée dans le sens de l'Histoire49».

Dans son rôle de défenseur de la non-figuration, Repentigny nẻ fut jamais un adversaire implacable de la figuration. Il rechercha plutôt l'affirmation d'un art créateur, indépendamment de la forme, et en suivit les tendances. L'ensemble de son travail critique, favorable à la non-figuration, se voulait un effort pour sortir cette forme d'art de la marginalisation. Pour contrer ces bien-pensants, ces «snobs» de la société comme il les appelle, qui devant "l'altérité» de l'art contemporain tentaient d'en brimer le développement au nom des valeurs traditionnelles "de conservation", Repentigny identifia la pratique de la non-figuration à une prise de la liberté dans le contexte duplessiste, et sa propre pratique critique existentialiste à un effort de constitution de cette altérité en devenir. Dans l'histoire des idées au Québec, sa pensée organisée et structurée proposant une libération individuelle et collective fondée sur le refus des conditionneménts totalitaires, fut assurément l'une des vigiles les plus exemplaires de l'insoumission et de l'insubordination intellectuelle.

L'aspect novateur, l'originalité de sa démarche intellectuelle imposa son oeuvre comme une critique paradigmatique, instauratrice d'une vision libertaire de l'homme dans sa société. 
1. François-Marc Gagnon, «Mimétisme en peinture contemporaine au Québec», in Peinture canadienne-française (débats). Conférences J.A. de Sève 11-12 édition augmentée, Presses de l'Université de Montréal, 1971, 1 i4 p., p. 39-60; François-Marc Gagnon, «Le Refus Global en son temps», in Ozias Leduc et Paul-Émile Borduas. Conférences J.A. de Sève 15-16, Presses de l'Université de Montréal, 1973, 152 p., p. 59-95.

2. Fernand Toupin, l'Atelier, cahier no. 15, 9 décembre 1980, interview et texte de Marcel Bélanger, Service des transcriptions et dérivés de la radio, Maison de Radio-Canada, 19 p.

3. R. de Repentigny, Manifeste des Plasticiens, 10 février 1955, publié hors commerce; reproduit in Jauran et les Premiers Plasticiens, Musée d'art contemporain, Montréal, 21 avril - 22 mai 1977, Editeur officiel du Québec, 1977, n p.

4. Repentigny publia aussi dans la revue Loisirs-Orientation (vol. 1, no. 1, à 16, de novembre 56 à avril 57), dans Arts et Pensée (vol. 3, no. 17) et dans Cahiers d'essai (vol. 1, no. 1).

5. Repentigny rédigea son premier article de critique d'art dans la Presse, «Deux peintres montréalais et un graveur de Toronto", le 25 octobre 1952.

6. R. de Repentigny, «La semaine des musées et les écoles», la Presse, 6 octobre 1956.

7. R. de Repentigny, "Des peintres qui ne voient pas à travers les fenêttres", la Presse, 22 novembre 1952.

8. R. de Repentigny, "Vitalité de l'art: la matière chante», la Presse, 24 avril 1954.

9. Wassily Kandinsky, Du spirituel dans l'art, Médiations, Denoël-Gonthier, 1969, 183 p.

10. R. de Repentigny; "Comment faut-il regarder une toile non-figurative», l'Autorité, ler mai 1954.

11. R. de Repentigny, Manifeste des Plasticiens, 10 février 1955, publié hors commerce; op. cit.

12. R. de Repentigny, "Quelques leçons de Matisse», la Presse, 6 novembre 1954.

13. R. de Repentigny, «Ne pas confondre les genres», la Presse, 5 septembre 1954.

14. $\dot{R}$. de Repentigny, «L'art enseigne l'habitude de la vérité», la Presse, 4 décembre 1954.

15. R. de Repentigny, Manifeste des Plasticiens, 10 février 1955, publié hors-commerce; $o p$. cit.

16. R. de Repentigny, «Romantisme et classicisme du XXe siècle», la Presse, 26 mars 1955.

17. Ibid.

18. R. de Repentigny, «Comment faut-il regarder une toile non-figurative», l'Autorité, ler mai 1954.

19. R. de Repentigny, «L'oeuvre doit avoir un destin «vrai»», la Presse, 24 décembre 1954.

20. R. de Repentigny, Manifeste des Plasticiens, 10 février 1955, publié hors commerce; op. cit.

21. R. de Repentigny, «Vivante profusion de Zadkine», la Presse, 26 juin 1954.

22. R. de Repentigny, «Pas le passé, ni l'avenir: le présent», la Presse, 26 juin 1954.

23. Ibid.

24. André Malraux, les Voix du silence, La galerie de la pléiade, Paris, 1953, 657 p.

25. R. de Repentigny, «Pour nous apprendre à voir», la Presse, 14 mai 1955.

26. Ibid.

27. R. de Repentigny, «L'art est aussi lien avec le monde», la Presse, 25 février 1956.

28. Ibid.

29. R. de Repentigny, "Vers la fin de l'individualisme en art", la Presse, 28 avril 1956.

30. R. de Repentigny, "Quand la peinture se colore de l'âpreté de la ville industrielle», la Presse, 12 mars 1957.

31. R. de Repentigny, «Pas d'art vrai sans progrès intérieur», la Presse,19 mars 1955.

32. Ibid.

33. R. de Repentigny, «Méditation sur l'actualité de l'art à Montréal», l'Autorité, 30 octobre 1954. 
34. R. de Repentigny, "Gare aux néo-académismes», la Presse, 19 novembre 1955.

35. R. de Repentigny, "Toutes les tendances dans le "groupe des peintres»", l'Autorité, 28 février 1953.

36. Pour plus d'informations, voir notre mémoire de maîtrise l'Oeuvre critique et plastique de Rodolphe de Repentigny, dép. d'Histoire de l'art, UQAM, 1983.

37. R. de Repentigny, "Les Plasticiens» et deux femmes-peintres»; la Presse, 9 novembre 1954.

38. R. de Repentigny, «Après les automatistes et les romantiques du surréalisme: les Plasticiens", l'Autorité, 6 novembre 1954.

39. R. de Repentigny, «Des révélations au Petit Salon d'Été», la Presse, 10 juillet 1954.

40. R. de Repentigny, «Romantișme et classicisme du XXe siècle», la Presse, 26 mars 1955.

41. R. de Repentigny, Manifeste des Plasticiens, 10 février 1955, publié hors commerce; op. cit.

42. André Malraux, les Voix du silence, op. cit.

43. R. de Repentigny, "Un cycle dans l'histoire de l'art», la Presse, 5 février 1955.

44. Ibid.

45. Ibid.

46. R. de Repentigny, «Deux grands maîtres de notre temps», la Presse, 31 juillet 1954.

47. Piet Mondrian, «Le home - la rue - la cité», Art d'aujourd'hui, vol. 1, no 5, décembre 1949 , n. p.

48. R. de Repentigny, "Un cycle dans l'histoire de l'art», la Presse, 5 février 1955.

49. R. de Repentigny, «Romantisme et classicisme du XXe siècle», la Presse, 26 mars 1955. 